\title{
Contemporary aspects in the prognosis of traumatic hyphemas
}

\author{
Dimitris Papaconstantinou' \\ Ilias Georgalas ${ }^{2}$ \\ Nikos Kourtis' \\ Eftimios Karmiris' \\ Chrysanthi Koutsandrea' \\ loannis Ladas' \\ Gerasimos Georgopoulos' \\ 'Department of Ophthalmology, \\ University of Athens, Athens, Greece; \\ 2"G Genimatas" Hospital, NHS, \\ Athens, Greece
}

\begin{abstract}
Purpose: The present study concerns traumatic hyphemas and their prognostic factors and signs. The aim of this study is to determine the prognostic factors and signs of traumatic hyphemas. Methods: During the last five years, 72 young individuals were hospitalized with the diagnosis of suffering a traumatic hyphema and were divided in three groups according to the extent of their hyphema. The first group concerns 38 patients with a small hyphema $3-4 \mathrm{~mm}$, the second group concerns 22 patients with moderate hyphema reaching the pupillary border, and the third group concerns 12 patients with a total hyphema.

Results: The hyphema was absorbed in 63 patients and the IOP was controlled with medical treatment after 3-24 days. However, surgical management was necessary for two patients. Finally, antiglaucomatous treatment was administered in seven patients with persistent high intraocular pressure.
\end{abstract}

Conclusions: The important clinical signs that determine the prognosis of such hyphemas are the size of hyphema, the blood color, recurrent hemorrhage, the absorption time, the increase of intraocular pressure, and blood staining of the cornea.

Keywords: traumatic hyphema, IOP rise, prognostic signs, blood staining of the cornea

\section{Introduction}

A hyphema is the presence of blood in the anterior chamber and the two most common causes are trauma of the globe and eye surgery. Other causes are iris neovascularization (eg, in diabetic retinopathy, central retinal vein occlusion, or chronic retinal detachment), juvenile xanthogranuloma, melanoma of the iris, myotonic dystrophy, leukemia, thrombopenia, von Willebrand disease, etc. ${ }^{1-3}$

The mean annual incidence of hyphemas is approximately 17 patients in 100,000, with a peak incidence between 10 and 20 years. The mean age of presentation is 25 years. ${ }^{1}$

In urban areas, two thirds of traumatic hyphemas are due to blunt ocular trauma and only one third due to traumatic rupture of the globe. ${ }^{1}$ Blunt ocular traumas caused by injury of the globe or peribulbar tissues and are followed by an anteroposterior compression of the globe and a simultaneous elongation of the equatorial area. This results in pressure on the anatomic components of the anterior segment, in rupture of iris or ciliary body's blood vessels and finally in hemorrhage in the anterior chamber (Figure 1). The hemorrhage may occupy the total volume of the anterior chamber, but usually occupies only a part of it, creating a level due to gravity effect. ${ }^{1-4}$ Secondary hemorrhage may occur because of blood clot dissolution and contraction of the traumatized vessels. ${ }^{5,6}$ Another mechanism of hyphema creation, in case of blunt ocular trauma, is the direct damage of blood vessels and the post-traumatic hypotony. ${ }^{1,2,4}$

\section{Methods}

This study investigates the factors that relate to the prognosis of the anterior chamber hyphemas caused by blunt ocular traumas. 
Seventy-two patients were included, they were treated at the 1st University Eye Clinic of Athens, Greece and their demographic characteristics are displayed in Table 1.

Two of the patients that were included in the study had the sickle cell trait and one patient was on anticoagulant medication.

At the baseline examination, a detailed history was taken about the specific circumstances under which the trauma took place and also a general medical history about other diseases (anemia, blood disorders, medications used, and liver or kidney disease) (Tables 2 and 3).

The eye examination included a detailed examination at the slit-lamp, intraocular pressure (IOP) measurement, fundus examination when it was possible (clear ocular media) with a +78 diopter lens or with the indirect ophthalmoscope. There was no use of the three-mirror Goldmann or any other type of goniolens during the initial examination in order to avoid any pressure on the globe and a secondary hemorrhage. We performed a gonioscopy after the hyphema was absorbed to check for the presence of a possible angle recession.

In certain cases, the patient underwent a computed tomography (CT) scan, magnetic resonance imaging (MRI) of the orbit, or a $\beta$-ultrasonography with special care taken not to exert pressure on the globe.

During the hospital treatment, patients had a daily slit-lamp examination and IOP measurement twice daily. According to the hyphema height the patients were divided in three groups: In the first group, 38 patients were included with a small hyphema (3-4 mm height; Figure 2); the second group had 22 patients with a moderate hyphema (Figure 3) reaching the pupillary border; the third group had 12 patients with a severe hyphema involving the total volume of the anterior chamber.

\section{Intervention results}

Treatment strategy comprises a supine position in bed with elevation of the head at an angle of $30^{\circ}-45^{\circ}$ in order to facilitate blood precipitation and absorption. In this way we contribute to the prevention of complications.

Medication in all patients encompasses topical steroid drops and a mild mydriatic. In certain patients in which an IOP elevation was observed, we used drops of a $\beta$-blocker and/or a

Table I Demographic characteristics

\begin{tabular}{ll}
\hline No. of patients & 72 \\
Age I5-47 & Mean age, 24.4 years \\
Sex & 58 men, I4 women \\
Race & 70 Caucasian, 2 non-Caucasian \\
\hline
\end{tabular}

Table 2 Causes of hyphema-related trauma

\begin{tabular}{ll}
\hline Affray/beating & 33 \\
Sport activities & 24 \\
Car accidents & 7 \\
Industrial accidents & 4 \\
Other causes & 4 \\
\hline
\end{tabular}

carbonic anhydrase inhibitor, and in significant IOP elevation we introduced a carbonic anhydrase inhibitor per os.

In the majority of the patients (63 patients; $87.5 \%$ ) treatment results were satisfying with adequate control of the IOP and resolution of the hyphema in a period of 3-24 days.

Secondary hemorrhage was observed the 3 rd day in one patient and the 4th day in two others. One of them had the sickle cell trait, another was on anticoagulant medication that had discontinued immediately after the occurrence of the hyphema, and the third patient suffered a moderate hyphema without any known predisposing factor.

Two patients in the 3rd group had an hyphema with a duration of more than 20 days and a sustained increased IOP, so they underwent surgery to evacuate the blood from the anterior chamber and to create a peripheral iridectomy.

Seven patients of the same group, after the absorption of the blood, suffered a persistent increase of the IOP above the higher normal limits and they were treated as open angle glaucoma patients. All of them were controlled medically except one patient who also had a vitreous hemorrhage. It was diagnosed as a ghost-cell glaucoma and underwent vitrectomy to control the elevated IOP. Among the patients that needed antihypertensive medication, gonioscopy revealed angle recession in three.

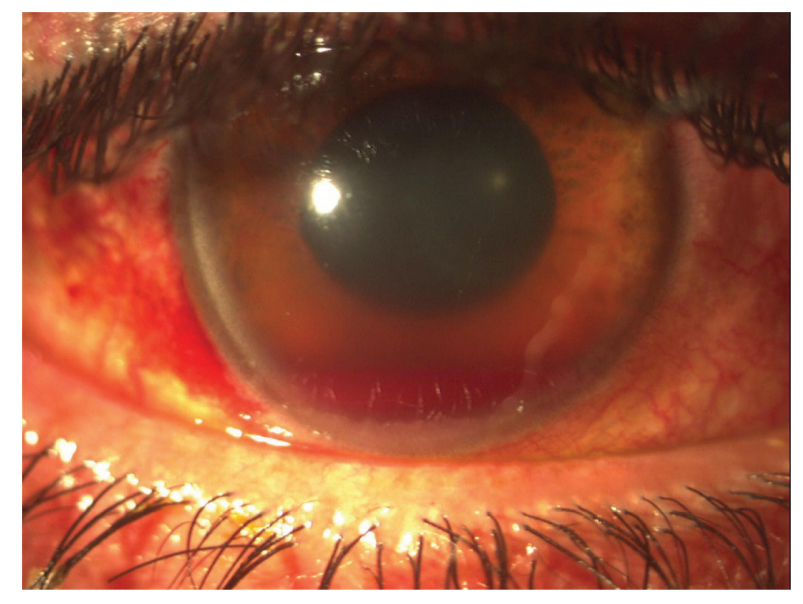

Figure I Patient with a moderate hyphema, iridoplegia, laceration of the pupillary sphincter at 9 o'clock and conjuctival hemorrhage at the site of globe injury. 


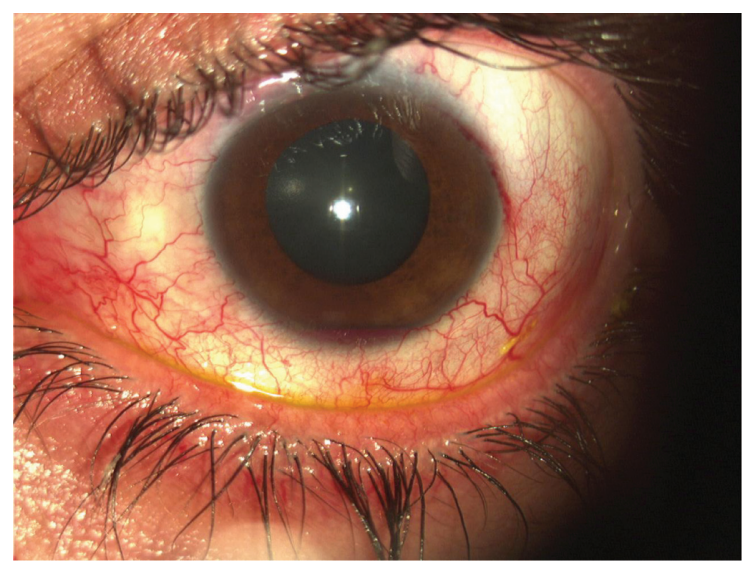

Figure 2 Patient with a small hyphema.

\section{Discussion}

The most important clinical signs that relate to the prognosis of traumatic hyphema, according to our experience and current literature ${ }^{1-21}$ are the following:

\section{The hyphema height}

Small hyphemas with a height of 3-4 mm, usually have a favorable course, which means that the anterior chamber hemorrhage is absorbed during the 1st week after the injury without any other complications. On the contrary, as far as it concerns the prognosis of the moderate and especially of the severe hyphemas, we should be quite skeptical. . $^{1,7}$

\section{Reoccurrence of hemorrhage}

Reoccurrence of hemorrhage is usually observed between the 3rd and 5th day and is a consequence of the normal blood clot dissolution that takes place at the site at which the blood vessel was traumatized. The secondary hemorrhage is usually heavier, occupies the total volume of the anterior chamber, and according to various sources is encountered with a frequency of $0.4 \%$ to $38 \% .1,5,6,11$ In our study, secondary hemorrhage occurred in three patients (4\%). Two were high risk patients for re-bleeding (sickle cell trait and anticoagulant medication). $)^{5,6,7,16}$

Table 3 Sport activities related to traumatic hyphemas

\begin{tabular}{ll}
\hline Tennis & 8 \\
Basketball & 5 \\
Water polo & 4 \\
Football & 4 \\
Volleyball & 3 \\
\hline
\end{tabular}

\section{Blood color}

Light red color is indicative of a continuous circulation of the aqueous humor and of an efficient oxygen supply in the anterior chamber. On the contrary, dark red or black color of the blood (due to the transformation of hemoglobin in methemoglobin) shows the discontinuation of the aqueous humor circulation and the lack of oxygen in the anterior chamber, which means the prognosis of hyphema must be made very cautiously. ${ }^{12}$

\section{IOP elevation}

Intraocular pressure elevation is the primary and most serious complication of hyphema because a major elevation of the IOP could cause corneal staining and optic nerve atrophy. ${ }^{10,13,16,17}$ The IOP fluctuation after an injury of the globe is as follows:

1. A moderate elevation $(<24 \mathrm{mmHg}$ ) immediately after the injury with a duration of 6-18 hours, due to mechanical obstruction of the trabecular meshwork from blood constituents and inflammatory debris. In addition, there can be a direct injury and a following edema of the trabecular meshwork.

2. Recovery of the IOP in normal values or even hypotony. The latter is observed from the 2nd until the 4th or 5th day and increases significantly the risk of a secondary hemorrhage. The cause of the hypotony is the reduction of the aqueous humor production due to ciliary body trauma.

3. Gradual increase of the IOP beginning the 5th-6th day that can reach high values. The reason for this phenomenon is dual: the return of the ciliary body function and the sustained obstruction of the aqueous humor circulation.

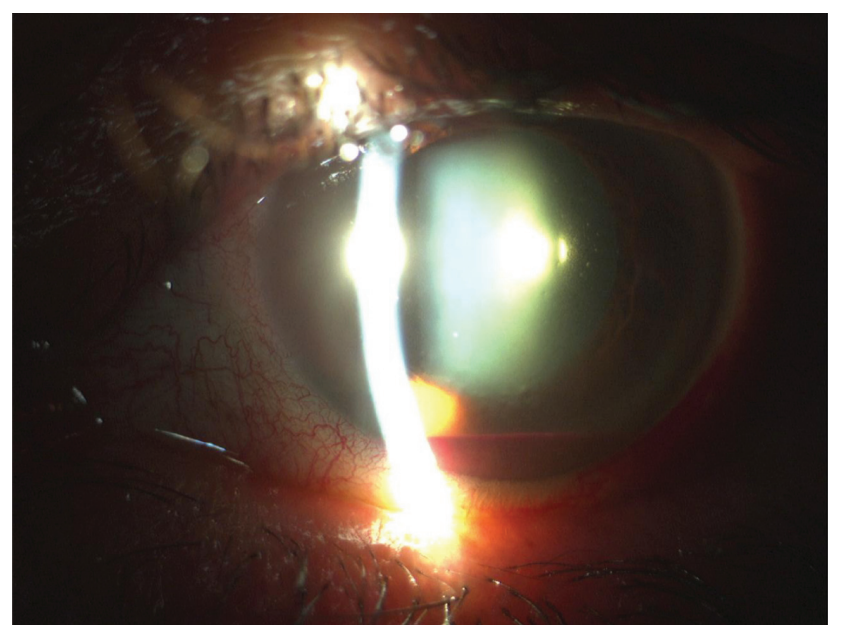

Figure 3 Patient with a moderate hyphema. 
4. A return of the IOP in normal values due to the absorption of the blood and the reinstitution of the trabecular meshwork function after approximately 4-18 days. Despite this, a secondary metatraumatic glaucoma may be established.

\section{Corneal blood staining}

A long standing hemorrhage of the anterior chamber along with a high IOP may cause blood constituents to enter the cornea and result in discoloration of the cornea with a yellow or light brown color. Other factors that contribute to this complication are the damage and transformation of the corneal endothelial cells and microerosions of the membrane of Descemet as a consequence of the trauma. ${ }^{14,20,21}$ Corneal blood staining may persist for a long period and its absorption starts from the periphery towards the centre of the cornea.

\section{Conclusions}

As a conclusion, the prognosis of the traumatic hyphema depends on the hyphema height, its color, the reoccurrence of the hemorrhage, the time that takes for the anterior chamber to clear the blood, and mostly on the IOP rise and the corneal blood staining. Patients with predisposing factors are associated with a higher risk of complications and should be more closely followed.

\section{Disclosure}

The authors report no conflicts of interest in this work.

\section{References}

1. Walton W, Von Hagen S, Grigorian R, Zarbin M. Management of traumatic hyphema. Surv Ophthalmol. 2002;47(4):297-334.

2. Brandt MT, Haug RH. Traumatic hyphema: a comprehensive review. J Oral Maxillofac Surg. 2001;59(12):1462-1470.

3. Edward WC, Layden WE. Traumatic hyphema. A report of 184 consecutive cases. Am J Ophthalmol. 1973;75(1):110-116.
4. Shingleton BJ, Hersh PS, Kenyon KR, editors. Eye Trauma. St. Louis, MO: Mosby; 1991; p. 104-116.

5. Witteman GJ, Brubaker SJ, Johnson M, Marks RG. The incidence of rebleeding in traumatic hyphema. Ann Ophthalmol. 1985;17(9): 525-526, 528-529.

6. Thomas MA, Parrish RK 2nd, Feuer WJ. Rebleeding after traumatic hyphemas. Arch Ophthalmol. 1986;104(2):206-210.

7. Lai WW, Edward DP, Tessler HH, Bhavnani VD. Risk factors for complications following traumatic hyphema. Arch Ophthalmol. 2001;119(11):1732.

8. Karim A, Laghmari M, Dahreddine M, et al. Hyphema with secondary hemorrhage: think about sickle cell disease. J Fr Ophtalmol. 2004;27(4):397-400.

9. Sankar PS, Chen TC, Grosskreutz CL, Pasquale LR. Traumatic hyphema. Int Ophthalmol Clin. 2002;42(3):57-68.

10. Layden WE. Traumatic glaucoma. Trans Am Acad Ophthalmol Otolaryngol. 1974;78(2):OP346-351.

11. Rahmani B, Jahadi HR, Rajaeefard A. An analysis of risk for secondary hemorrhage in traumatic hyphema. Ophthalmology. 1999;106(2): 380-385.

12. Schein OD, Hibberd PL, Shingleton BJ, Kunzweiler T, Frambach DA, Seddon JM, Fontan NL, Vinger PF. The spectrum and burden of ocular injury. Ophthalmology. 1988;95(3):300-305.

13. Hitchings R. Traumatic glaucoma. J Glaucoma. 2001;10(5): 436-439.

14. Slingsby JG, Forstot SL. Effect of blunt trauma on the corneal endothelium. Arch Ophthalmol. 1981;99(6):1041-1043.

15. Yu T, Dahan E, Yin ZQ, Levitz LM. Use of an anterior chamber maintainer in the surgical management of traumatic hyphaemas. Clin Experiment Ophthalmol. 2008;36(3):206-208.

16. Ashaye AO. Traumatic hyphaema: a report of 472 consecutive cases. BMC Ophthalmol. 2008;8:24.

17. Sihota R, Kumar S, Gupta V, Dada T, Kashyap S, Insan R, Srinivasan G. Early predictors of traumatic glaucoma after closed globe injury: trabecular pigmentation, widened angle recess, and higher baseline intraocular pressure. Arch Ophthalmol. 2008;126(7):921-926.

18. Pollock T, Laliberté I, Wu J, Lyons C. Traumatic hyphema and immune thrombocytopenic purpura: late rebleeds associated with low platelet count. Can J Ophthalmol. 2008;43(6):717.

19. Albiani DA, Hodge WG, Pan YI, Urton TE, Clarke WN. Tranexamic acid in the treatment of pediatric traumatic hyphema. Can J Ophthalmol. 2008;43(4):428-431.

20. Pong J, Lai J. Effect on corneal endothelial cell count of traumatic microhyphaema and hyphaema. Acta Ophthalmol. 2008 Aug 11. [Epub ahead of print].

21. Kloek C, Brauner S, Chen TC. Corneal blood staining after traumatic hyphema. J Pediatr Ophthalmol Strabismus. 2007;44(4):256. 